\title{
An Iterative Descent Method for Predicting The Compressive Cement Strength Estimated Parameters
}

\author{
Adeline Goullieux, Mhand Hifi, Shohre Sadeghsa \\ EPROAD, Université de Picardie Jules Verne \\ 7 rue du Moulin Neuf, 80000 Amiens, France \\ Emails: Adeline.goullieux@u-picardie.fr, hifi@u-picardie.fr, shohre.sadeghsa@u-picardie.fr
}

\begin{abstract}
In this paper, we propose an iterative descent method to predict compressive cement strength estimated parameters for lime and cement as coating substances. We first propose a formal description of the problem by using a mathematical model that is based upon a series of equations. The aforementioned equations are related to both the ratio of quantity of water to quantity of coating substance and the ratio of quantity of straw to quantity of coating substance. Second, we propose to solve the model by applying a gradient descent method. It is applied for reaching results closest to the data gathered from a real experimental studies conducted on the coating of flax straw before incorporating them into a cement matrix. The experimental part shows that the proposed model is capable to predict The the estimation of the parameters necessary for such study.
\end{abstract}

Index Terms-Prediction; Optimization; Gradient descent.

\section{INTRODUCTION}

T HE MOST important mechanical property of a concrete is it's compressive strength. Compressive strength test is a commonly used experiment to measure the characteristics of the cement properties. In this study the experiment is conducted in the laboratory of IMAP on the coating of flax straw before their incorporation into a cement matrix. During the experiment, the ratio of quantity of water to quantity of Coating Substance (noted CS) and the ratio of the quantity of straw to quantity of CS varied.

Measured characteristics are density, water absorption capacity and decrease in water absorption capacity (comparison with untreated straws) of straws and flexural strength, compressive strength, density, dimensional variation on drying and extreme dimensional variation of composites. The objective of the experiment is to have high mechanical properties and low dimensional variation on the formulated product.

Several studies proposed soft computing-based approaches to predict the mechanical strength of concrete. Generally, these approaches tend to design models based upon linear and / or nonlinear regression equations (cf., Snell, et al. [1]).

During practical sessions, we noticed that this experiment is time consuming and expensive as it needs to recruit people to perform the experiments. In order to overcome these difficulties, often encountered in these studies, we propose a mathematical model for predicting results related to the principal parameters of the study.

The rest of the paper is organized as follows. Section II describes a brief overview of the related studies. In section III, the parameters of the experiment is described, where a brief representation of the data used is provided in section IV. Section V shows how linear regression-based method can be applied for the considered study. Finally, the proposed equations are presented in section VI and the last section VII concludes the paper by summarizing the contents of the study and future studies.

\section{AN OVERVIEW OF THE LITERATURE}

Compressive Cement Strength Test (namely CCST) is the most important test for quality control which conventionally takes 28 days of experiments (for more details the reader can be referred to de Siqueira Tango [2]).

Generally there are two ways to perform CCST: (i) the first way can be achieved by considering the experimental test, where Accelerated Atrength test methods (namely AST) is applied and, (ii) the second way based on predicting the variables-values using soft computing-based methods.

CCST by itself is time consuming and therefore expensive for industries. Considering the importance of CCST, we decided to simulate the experiment by proposing mathematical formulations. Indeed, we mainly propose equations in this study that are able to simulate the variables of CCST.

We note that there are several studies that focus on the soft computing-based methods, including regressions and neural networks. However regression methods seems popular and less complicated to tackle when compared to the other studies (cf., de Siqueira Tango [2] and, Snell, et al. [1]).

Behavior of these materials by itself depends on several chemical and physical parameters that affect each other in nature. Therefore, detecting and optimizing these materials is not the most efficient method. Thus, both statistical and analytical models with the use of soft computing-based approaches, including regression analysis seems a promising way to the Compressive Cement Strength Prediction Problem (noted CCSPP).

The optimization of concrete (and generally composites) concerns selecting values for the constituent parameters. Baykasoglu et al. [4] proposed a two-step approach for multi objective optimization of high-strength concretes (namely HSCs). In their study, they applied a regression analysis, a neural networks and a gen expression programming to predict HSCs parameters in the first step. The first step established equations that represent concrete characteristic in terms of its components. According to these equations, a multiobjective optimization model has been proposed that is solved by using a genetic algorithm; that is the second step of their approach.

In Baykasoglu et al. [3] another way to tackle the CCSPP has been proposed. Indeed, the authors proposed a gene 
expression programming, neural networks and stepwise regression analysis as soft computing techniques.

Herein, we aim to simulate CCST by mathematical equations. The equations will return the parameters that needs to be varied in a real experiment. Results of the equations obtained in this study can be replaced with carried experiment.

\section{COMPRESSIVE CEMENT STRENGTH TEST PARAMETERS}

In this study we considered two different experiments:

1) The first experiment. The objective is to predict the ratio of quantity of water to quantity of CS and the ratio of quantity of straw to quantity of CS for lime by having the density, water absorption capacity and decrease in water absorption capacity (comparison with untreated straws) of straws and flexural strength, compressive strength, density, dimensional variation on drying and extreme dimensional variation of composites. This experiment will results two equation where each of this equations is used to predict a ratio.

2) The second experiment. In addition to the first experiment, the second experiment will calculate the two ratios related to the cement.

Table I reports the parameters used in this study. These parameters are considered in both experiments for Lime and Cement.

TABLE I: Design variables

\begin{tabular}{l|l} 
Symbol & Parameter \\
\hline $\mathrm{X} 1$ & Shive water absorbance diminution (\%) \\
$\mathrm{X} 2$ & Shive water absorbance $(\%)$ \\
$\mathrm{X} 3$ & Shive bulk density $(\mathrm{kg} / \mathrm{m} 3)$ \\
$\mathrm{X} 4$ & Composite Rf $(\mathrm{Mpa})$ \\
$\mathrm{X} 5$ & Composite Rc (MPa) \\
$\mathrm{X} 6$ & Composite bulk density $(\mathrm{kg} / \mathrm{m} 3)$ \\
$\mathrm{X} 7$ & Composite DS $(\mathrm{mm} / \mathrm{m})$ \\
$\mathrm{X} 8$ & Composite EDV $(\mathrm{mm} / \mathrm{m})$ \\
\hline $\mathrm{Y} 1$ & Shive/Coating substance $(\mathrm{S} / \mathrm{CS})$ \\
$\mathrm{Y} 2$ & Water/Coating Substance $(\mathrm{W} / \mathrm{CS})$ \\
\hline
\end{tabular}

Therefore, using symbols introduced in Table I, on the one hand, for the first experiment the parameters are represented by the set $\{\mathrm{X} 1, \mathrm{X} 2, \mathrm{X} 3, \mathrm{X} 4, \mathrm{X} 5, \mathrm{X} 6, \mathrm{X} 7, \mathrm{X} 8\}$ and $\mathrm{Y} 1$ denotes the variable to predict for Lime. On the other hand, for the second equation the parameters are characterized with the set $\{\mathrm{X} 1, \mathrm{X} 2, \mathrm{X} 3, \mathrm{X} 4, \mathrm{X} 5, \mathrm{X} 6, \mathrm{X} 7, \mathrm{X} 8\}$ and $\mathrm{Y} 2$ is the predicted variable. In the second experiment the similar parameters are selected using data of Cement.

\section{DATA COLLECTION}

Practical experiments are conducted in IMAP Laboratory, where 123 experiments were conducted on the coating substances including: (i) 60 experiments on Lime, (ii) 3 on the non treated material and, (iii) 60 experiments on Cement.

In the experiment, for each $\mathrm{CS}$, the ratio of quantity of water to quantity of $\mathrm{CS}$ is varied in the interval $\{0.33,0.5,0.75$, 1 ) while the ratio of the quantity of straw to quantity of CS is varied in interval $\{0.5,0.751,2\}$.
For each CS the measured parameters are shive water absorbance (\%), shive bulk density ( $\mathrm{kg} / \mathrm{m} 3)$, composite $\mathrm{Rf}$ (Mpa), composite Rc (MPa), composite bulk density $(\mathrm{kg} / \mathrm{m} 3)$, composite DS $(\mathrm{mm} / \mathrm{m})$, composite EDV $(\mathrm{mm} / \mathrm{m})$. Finally, the amount of shive water absorbance diminution (\%) is mathematically calculated using the provided data from the experiments.

We note that the goal of the experiment is to preserve high mechanical properties and low dimensional variation on the formulated coating substances. Average of the data for each experiment is summarized in Table III

\section{REGRESSION ANALYSIS}

There are several studies in the literature that implement various algorithms to optimize gradient descent as one of the most popular optimization methods.(e.g. Bengio [6])

The gradient descent my be distinguished with its three variants: (i) batch gradient descent, (ii) stochastic gradient descent and, (iii) mini-batch gradient descent. The difference between these varaints lies in the amount of the data.

Herein, as mentioned in below, data is expensive and collecting the data is time consuming. Therefore, the amount of available data are limited.

Batch gradient descent calculates the gradient for the whole data set. Therefore, dealing with large dataset, batch gradient descent performs very slowly. Because the number of data are limited in this study, we can safely use the batch gradient descent (for more details, the reader can refer to Ruder [5].

\section{A. Gradient descent}

One of the most applied methods in the literature, to perform optimization and prediction, we can cite the gradient descent; that is due to its simplicity and applicability for several applications and problems to solve.

In what follows, the aim is to establish linear equations to predict CCST parameters. Indeed, the proposed linear model may be formulated as follows:

$$
P(\theta)=\theta_{0}+X \theta
$$

In equation (1), $\theta_{0}$ refers to a constant value, $X$ deotes the parameters of the model and $\theta$ is the coefficient of the parameters. The objective function is defined as the average summation of the square prediction errors for the linear regression. Differently stated, the objective function is also referred as the following cost function:

$$
C(\theta)=\frac{1}{2 m} \sum_{i=1}^{m}\left(P(\theta)_{i}-Y_{i}\right)^{2},
$$

where $\mathrm{C}(\theta)$ represents the cost function for each $\theta, m$ denotes the number of the training examples and $Y$ is the actual value of the variable; that is predicted using the cost function.

The gradient descent tries to minimize the objective function using an iterative procedure, where the coefficients of the 
parameters of the model are updated by using the following equation:

$$
\theta_{j}:=\theta_{j}-\frac{\lambda}{m} \sum_{i=1}^{m}\left(P(\theta)_{i}-Y_{i}\right) X_{i}
$$

At each iteration of the gradient descent, $\theta$ will be updated to the opposite direction of the gradient of the objective function. The parameter $\lambda$ determines the learning rate. It determines step sizes to reach a local minimum. In other words, at each step we move to the direction of the slope of the objective function towards the bottom of a slope, until a local minimum is reached.

\section{B. Principal analysis}

Data described and explained in section IV are analyzed by using the following procedure:

1) Shuffling data.

As described in section IV, data are organized during experimental test. Thus, we shuffled the data to avoid miscalculations and computational errors. The shuffling is performed in a way that only the order of the data will be permuted randomly not the parameters of the experiments.

2) Separate dataset into training set and test set:

Data is separated into two groups: the training set and the test set. Shuffling the data in the previous step is necessary so that there will be no difference in the quality of the data in training and test set.

Statistical analysis will be performed in the training set and the quality of the method will be examined in the test set. Note that the size of the test set is setting equal to $30 \%$ of the data and the rest (i.e, $70 \%$ of data) represents the size of the test set.

3) Mean normalizing the features (average and standard deviations):

Measured data from CCST has various ranges and thus there is a great difference by order of the magnitude in these features. Table II represents both minimum and maximum values for each parameter. As in Table II, the range of the values are diversified (i.e, one parameter can be 100 times bigger than the other). In this case, scaling the features by mean normalization will drive the gradient descent-based algorithm to converge. Therefore, in order to perform gradient descent on the data, it is advised to mean normalize the data as follows:

$$
\frac{X-\mu}{\sigma}
$$

where $X$ denotes the value of the parameter to be normalized, $\mu$ and $\sigma$ represent the average and the standard deviations, respectively. Note that $\mu$ and $\sigma$ are calculated once on the training set and then they applied to normalize the test set.

4) Randomly initializing $\theta$ :

The parameter $\theta$ is the coefficient of the parameters that described in section V-A. Before applying the gradient descent, $\theta$ should be initialized. Herein, is is randomly initialized in the interval $[0,1]$.

5) Optimizing $\theta$ with the gradient descent algorithm: By defining the two parameters (number of iterations and learning rate), the gradient descent algorithm can be applied on the data. One way to verify if the gradient descent algorithm works correctly, it suffies to calculate the cost function and to verify if the value related to the average square of the prediction error as the cost function is decreasing at each iteration.

6) Prediction equation:

After normalizing test set data, using the average and standard deviations of the training set, predicting the variables may be computed as follows:

$$
Y=X \theta
$$

where $X$ denotes the normalized data from the test set and $Y$ is that corresponding to the predicted value.

Of course, for more comprehensive documentary and introductory, the reader can refer to $\mathrm{Ng}$ [7].

\section{Computational Results}

The data are analysed using GNU Octave, version 5.2.0 on a mac OS Catalina with $2.3 \mathrm{GHz}$ Intel Core i5 processor. Parameters tuning of the gradient descent procedure are considered as follows: (i) learning rate $\Lambda$ is set to 0.01 and (ii) the number of iterations is set to 50000 . Note that there are 60 data for each experiments. Thus, the computational time is negligible (less than a minute).

The prediction equations for the two experiments on Lime are provided as follows (cf., Equation 6 and Equation 7):

$\mathrm{Y} 1=0.86-0.05 \mathrm{X} 1+0.05 \mathrm{X} 2-0.20 \mathrm{X} 3+0.01 \mathrm{X} 4-0.26 \mathrm{X} 5+0.23 \mathrm{X} 6-0.01 \mathrm{X} 7+0.29 \mathrm{X} 8$

and

$\mathrm{Y} 2=1.01-0.05 \mathrm{X} 1+0.05 \mathrm{X} 2-0.21 \mathrm{X} 3+-0.07 \mathrm{X} 4-0.87 \mathrm{X} 5+0.90 \mathrm{X} 6-0.01 \mathrm{X} 7-0.27 \mathrm{X} 8$

The same procedure is applied for Cement, where the followings equations (Equation 8 and Equation 9) are used:

$\mathrm{Y} 1=0.92-0.13 \mathrm{X} 1+0.06 \mathrm{X} 2-0.22 \mathrm{X} 3+-0.07 \mathrm{X} 4+0.12 \mathrm{X} 5+-0.19 \mathrm{X} 6+0.13 \mathrm{X} 7+-0.08 \mathrm{X} 8$

and

$\mathrm{Y} 2=1.11-0.05 \mathrm{X} 10.02 \mathrm{X} 2-0.32 \mathrm{X} 3+0.05 \mathrm{X} 4+0.52 \mathrm{X} 5+-0.24 \mathrm{X} 6+0.04 \mathrm{X} 7-0.41 \mathrm{X} 8$

The performance of prediction via gradient descent algorithm to predict variables of CCST is depicted in Figure 1, Figure 2, Figure 3 and Figure 4. From the figures, one can observe what follows:

- First, the gradient descent can provide interesting predictions for the experiments

- Second, the errors of the proposed analysis may be due to the possible human errors in the data set and / or interactions between parameters of the study. We hope that the latter can be faced by applying neural network or logistics regressions instead of gradient descent. 


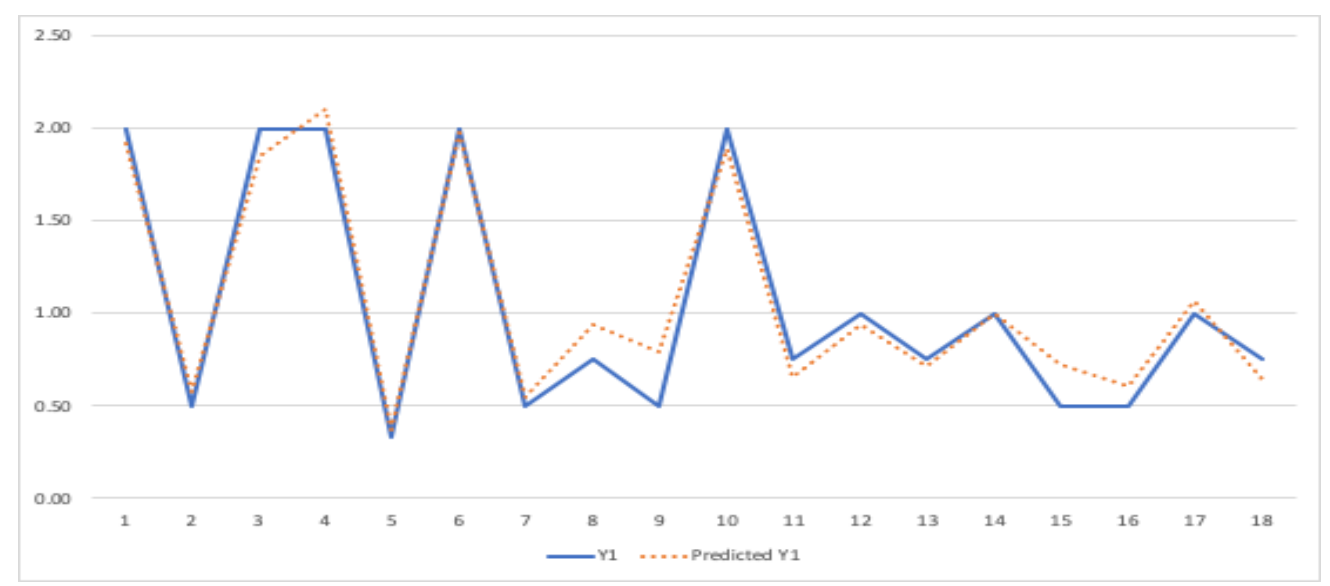

Fig. 1: Prediction of ratio of shive to coating substance $Y 1$ versus ratio of shive to coating substance for the test data for Lime

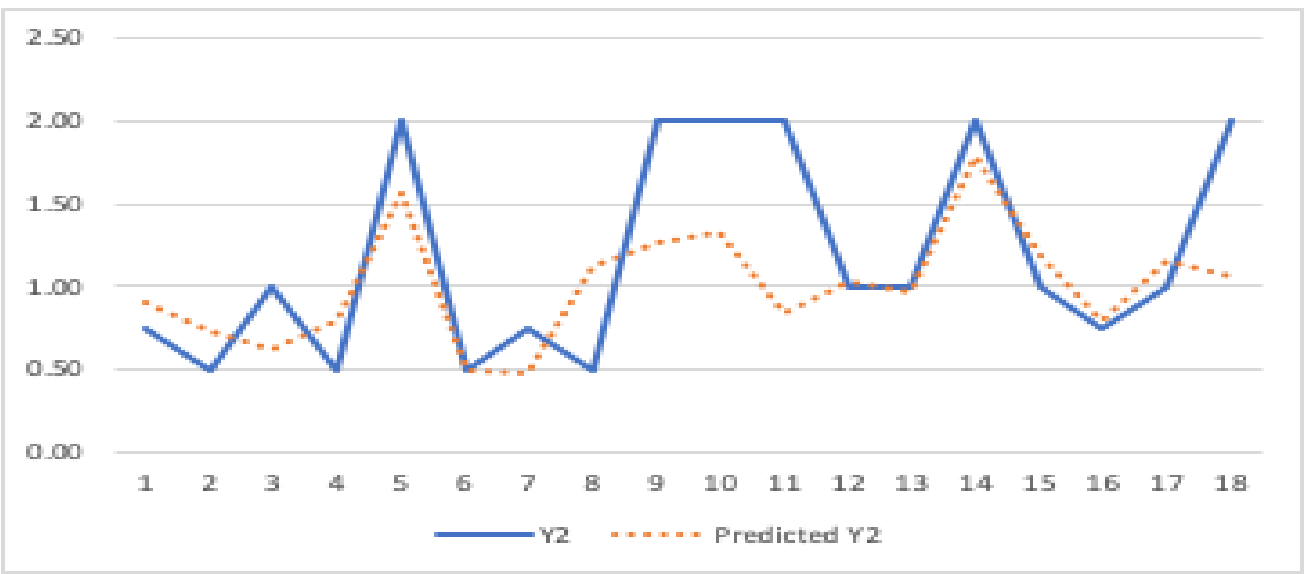

Fig. 2: Prediction of ratio of water to coating substance (Y2) VS ratio of water to coating substance for the test data for Lime

- Third, the average errors reaching the predictions related to the ratio of shive to coating substance and water to coating substance are equal to -0.02 and 0.17 for Lime and, equal to 0.00 and 0.10 for Cement accordingly.

- Fourth and last, the learning rate is set to 0.01 and the number of iterations is set to 60000. As the number of training set is less than 100 , the runtime is not significant (less than a minute). Finally, the established equations are parameterized using descriptions in Table I.

\section{CONCLUSION}

In this study we proposed mathematical equations to simulate the Compressive Cement Strength Test referred as CCST. Such a problem is the most important test in quality control for the industries. In practice, CCST takes 28 days and it is expensive to provide the final results. Therefore, this paper proposed an approach that is able to predict the parameters of the experiment. In order to establish efficient values for the parameters, a gradient descent-based algorithm has been proposed, where linear equations were considered. For future research, several directions of research can be considered. Indeed, first, neural networks can be adapted to predict some parameters and so, in some cases they can be applied for providing tight estimations. Second, the hybridization between neuronal networks and operational research technics can be envisaged for achieving better predictions of the parameters.

\section{REFERENCES}

[1] Snell, L. M., Van Roekel, J.,\& Wallace, N. D. (1989). Predicting early concrete strength. Concrete International, 11(12), 43-47.

[2] de Siqueira Tango, C. E. (1998). An extrapolation method for compressive strength prediction of hydraulic cement products. Cement and concrete research, 28(7), 969-983.

[3] Baykasoğlu, A., Dereli, T., \& Tanış, S. (2004). Prediction of cement strength using soft computing techniques. Cement and concrete research, 34(11), 2083-2090.

[4] Baykasoglu, A., Oztas, A., \& Ozbay, E. (2009). Prediction and multiobjective optimization of high-strength concrete parameters via soft computing approaches. Expert Systems with Applications, 36(3), 61456155.

[5] Ruder, S. (2016). An overview of gradient descent optimization algorithms. arXiv preprint arXiv:1609.04747.

[6] Bengio, Y., Boulanger-Lewandowski, N., \& Pascanu, R. (2013, May). Advances in optimizing recurrent networks. In 2013 IEEE International Conference on Acoustics, Speech and Signal Processing (pp. 86248628). IEEE.

[7] Ng, A. (2018). Machine Learning. Stanford University. línea]. Available: https://www. coursera. org/learn/machine-learning.[Último acceso: 14 apr 2017]. 
TABLE II: Range of parameters

\begin{tabular}{llllllllll} 
Coating substance $(\mathrm{CS})$ & Range & $\mathrm{X} 1(\%)$ & $\mathrm{X} 2(\%)$ & $\mathrm{X} 3(\mathrm{~kg} / \mathrm{m} 3)$ & $\mathrm{X} 4(\mathrm{Mpa})$ & $\mathrm{X} 5(\mathrm{MPa})$ & $\mathrm{X} 6(\mathrm{~kg} / \mathrm{m} 3)$ & $\mathrm{X} 7(\mathrm{~mm} / \mathrm{m})$ & $\mathrm{X} 8(\mathrm{~mm} / \mathrm{m})$ \\
\hline Lime & Min & 36.43 & 47.93 & 129.70 & 0.14 & 0.63 & 583.63 & 2.40 & 1.31 \\
Lime & Max & 77.66 & 136.40 & 298.50 & 2.57 & 9.56 & 1047.93 & 8.17 & 8.65 \\
Cement & $\min$ & 38.23 & 46.81 & 130.60 & 0.16 & 0.31 & 556.09 & 0.00 & 3.14 \\
Cement & $\max$ & 78.18 & 132.54 & 340.75 & 3.28 & 7.41 & 1099.49 & 7.66 & 6.76 \\
\hline
\end{tabular}

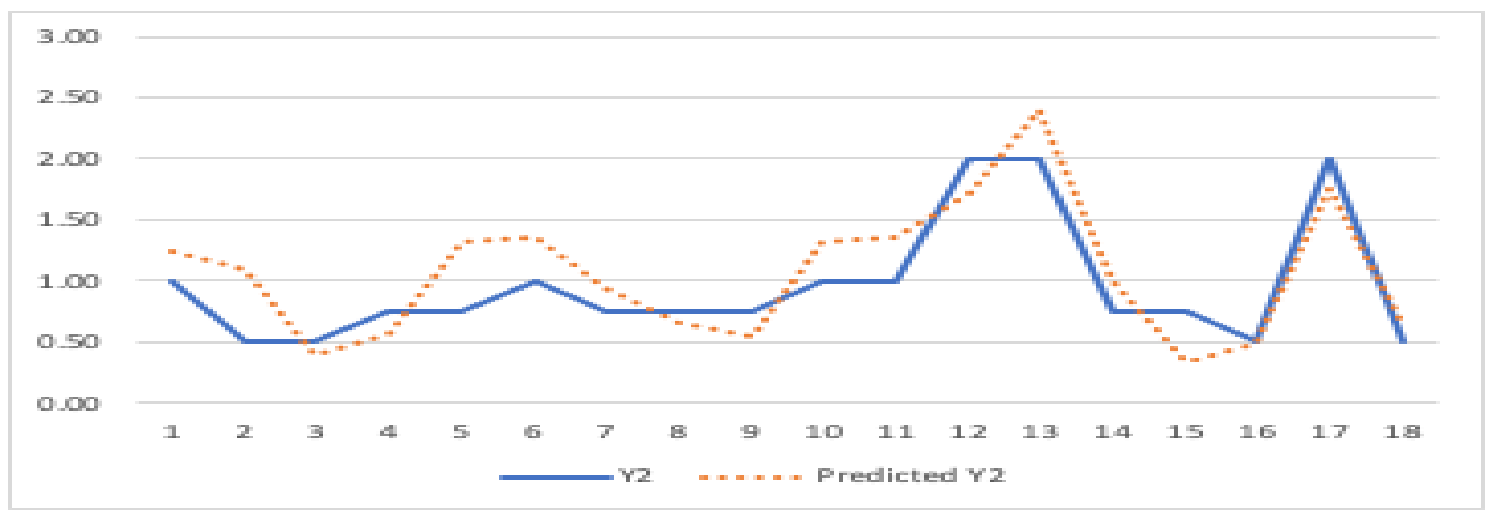

Fig. 3: Prediction of ratio of water to coating substance (Y2) VS ratio of water to coating substance for the test data for Cement

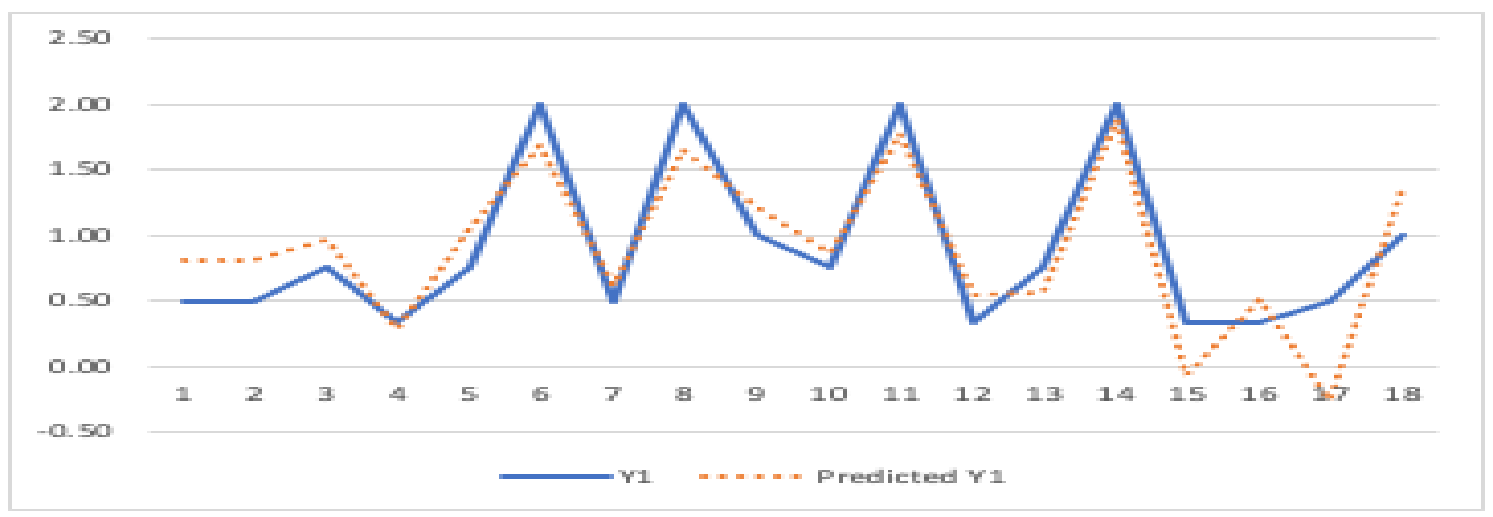

Fig. 4: Prediction of ratio of water to coating substance (Y1) VS ratio of water to coating substance for the test data for Cement 
TABLE III: Average data for each experiment

\begin{tabular}{|c|c|c|c|c|c|c|c|c|c|c|}
\hline Coating substance (CS) & Y1 (S/CS) & $\mathrm{Y} 2(\mathrm{~W} / \mathrm{CS})$ & $\mathrm{X} 1(\%)$ & X2 (\%) & $\mathrm{X} 3(\mathrm{~kg} / \mathrm{m} 3)$ & X4 (Mpa) & X5 (MPa) & $\mathrm{X} 6(\mathrm{~kg} / \mathrm{m} 3)$ & $\mathrm{X} 7(\mathrm{~mm} / \mathrm{m})$ & $\mathrm{X} 8(\mathrm{~mm} / \mathrm{m})$ \\
\hline Lime & 0.33 & 0.50 & 66.47 & 256.75 & 71.95 & 1.06 & 6.30 & 819.60 & 4.57 & 2.70 \\
\hline Lime & 0.33 & 0.75 & 60.76 & 261.10 & 84.20 & 1.86 & 8.89 & 1025.34 & 3.15 & 2.24 \\
\hline Lime & 0.33 & 1.00 & 67.97 & 295.13 & 68.72 & 1.79 & 4.88 & 911.35 & 4.81 & 1.95 \\
\hline Lime & 0.33 & 2.00 & 66.39 & 290.80 & 72.12 & 0.28 & 0.67 & 705.04 & 4.50 & 1.49 \\
\hline Lime & 0.50 & 0.50 & 64.46 & 217.43 & 76.25 & 0.55 & 1.77 & 643.55 & 4.57 & 2.99 \\
\hline Lime & 0.50 & 0.75 & 65.80 & 220.68 & 73.38 & 0.83 & 2.79 & 685.20 & 4.06 & 3.02 \\
\hline Lime & 0.50 & 1.00 & 65.59 & 218.68 & 73.84 & 1.53 & 6.39 & 914.18 & 3.25 & 2.85 \\
\hline Lime & 0.50 & 2.00 & 65.98 & 218.68 & 72.99 & 0.83 & 2.05 & 734.88 & 6.11 & 2.51 \\
\hline Lime & 0.67 & 0.50 & 61.14 & 211.98 & 83.38 & 1.01 & 2.23 & 696.17 & 5.64 & 3.35 \\
\hline Lime & 0.67 & 0.75 & 55.93 & 194.02 & 94.56 & 1.01 & 3.04 & 702.38 & 4.65 & 2.76 \\
\hline Lime & 0.67 & 1.00 & 66.40 & 208.57 & 72.08 & 1.72 & 3.64 & 779.18 & 3.89 & 2.71 \\
\hline Lime & 0.67 & 2.00 & 61.57 & 196.97 & 82.46 & 2.41 & 5.00 & 836.20 & 3.17 & 2.28 \\
\hline Lime & 1.00 & 0.50 & 55.57 & 184.07 & 95.32 & 0.59 & 1.46 & 651.26 & 6.29 & 4.68 \\
\hline Lime & 1.00 & 0.75 & 58.73 & 160.58 & 88.55 & 0.55 & 1.76 & 676.64 & 5.29 & 4.09 \\
\hline Lime & 1.00 & 1.00 & 57.80 & 170.38 & 90.54 & 0.79 & 2.33 & 686.95 & 5.03 & 3.65 \\
\hline Lime & 1.00 & 2.00 & 58.27 & 173.57 & 89.54 & 0.87 & 2.60 & 779.21 & 4.57 & 2.80 \\
\hline Lime & 2.00 & 0.50 & 46.76 & 140.00 & 114.22 & 0.19 & 0.83 & 602.45 & 6.68 & 8.22 \\
\hline Lime & 2.00 & 0.75 & 49.55 & 134.87 & 108.25 & 0.20 & 0.85 & 625.43 & 6.32 & 6.90 \\
\hline Lime & 2.00 & 1.00 & 48.82 & 130.93 & 109.81 & 0.22 & 0.95 & 591.77 & 6.28 & 7.53 \\
\hline Lime & 2.00 & 2.00 & 43.83 & 132.43 & 120.53 & 0.28 & 1.06 & 693.85 & 5.85 & 6.22 \\
\hline Cement & 0.33 & 0.50 & 71.48 & 318.27 & 61.19 & 0.55 & 1.29 & 810.66 & 6.09 & 3.76 \\
\hline Cement & 0.33 & 0.75 & 70.43 & 322.43 & 63.44 & 0.90 & 1.97 & 907.19 & 5.01 & 3.55 \\
\hline Cement & 0.33 & 1.00 & 71.34 & 328.15 & 61.48 & 1.10 & 2.98 & 933.54 & 5.01 & 3.26 \\
\hline Cement & 0.33 & 2.00 & 66.44 & 300.75 & 72.01 & 1.58 & 6.34 & 978.46 & 4.58 & 3.32 \\
\hline Cement & 0.50 & 0.50 & 64.47 & 233.48 & 76.22 & 0.67 & 1.59 & 761.58 & 5.04 & 3.86 \\
\hline Cement & 0.50 & 0.75 & 69.20 & 240.23 & 66.08 & 0.67 & 1.74 & 822.90 & 4.72 & 3.59 \\
\hline Cement & 0.50 & 1.00 & 65.88 & 234.35 & 73.21 & 1.33 & 2.21 & 840.21 & 5.21 & 3.43 \\
\hline Cement & 0.50 & 2.00 & 61.89 & 255.72 & 81.77 & 2.34 & 6.47 & 1076.02 & 2.72 & 3.51 \\
\hline Cement & 0.67 & 0.50 & 60.09 & 201.40 & 85.63 & 0.73 & 1.50 & 737.36 & 5.70 & 5.87 \\
\hline Cement & 0.67 & 0.75 & 57.43 & 197.65 & 91.33 & 1.10 & 2.75 & 748.96 & 5.42 & 5.19 \\
\hline Cement & 0.67 & 1.00 & 62.59 & 203.33 & 80.26 & 1.20 & 2.97 & 732.15 & 4.17 & 4.25 \\
\hline Cement & 0.67 & 2.00 & 61.54 & 198.97 & 82.52 & 2.45 & 6.53 & 916.17 & 3.87 & 4.12 \\
\hline Cement & 1.00 & 0.50 & 54.69 & 164.27 & 97.22 & 0.37 & 0.85 & 635.59 & 6.57 & 6.05 \\
\hline Cement & 1.00 & 0.75 & 58.84 & 165.83 & 88.31 & 0.49 & 1.20 & 614.77 & 6.41 & 6.00 \\
\hline Cement & 1.00 & 1.00 & 57.44 & 163.78 & 91.32 & 0.54 & 1.33 & 685.74 & 5.47 & 4.83 \\
\hline Cement & 1.00 & 2.00 & 57.62 & 158.47 & 90.93 & 1.22 & 2.55 & 748.84 & 5.08 & 3.98 \\
\hline Cement & 2.00 & 0.50 & 45.54 & 140.27 & 116.85 & 0.20 & 0.40 & 605.68 & 7.45 & 6.14 \\
\hline Cement & 2.00 & 0.75 & 40.35 & 136.27 & 127.99 & 0.26 & 0.50 & 596.42 & 6.95 & 6.21 \\
\hline Cement & 2.00 & 1.00 & 42.39 & 132.63 & 123.60 & 0.25 & 0.52 & 591.47 & 6.09 & 4.77 \\
\hline Cement & 2.00 & 2.00 & 46.68 & 133.28 & 114.40 & 0.37 & 0.83 & 576.58 & 5.65 & 4.26 \\
\hline
\end{tabular}

